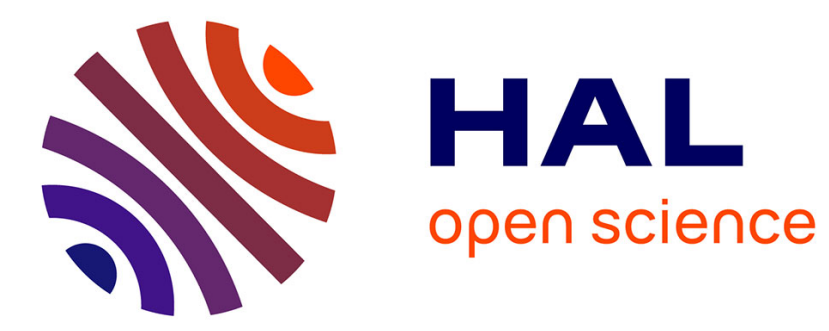

\title{
Le simulateur automatisé BirthSIM pour la formation des obstétriciens
}

Richard Moreau, Minh Tu Pham, Xavier Brun, Tanneguy Redarce, Olivier

Dupuis

\section{- To cite this version:}

Richard Moreau, Minh Tu Pham, Xavier Brun, Tanneguy Redarce, Olivier Dupuis. Le simulateur automatisé BirthSIM pour la formation des obstétriciens. Journal Européen des Systèmes Automatisés (JESA), 2011, 45 (4-6), pp.415-435. 10.3166/jesa.45.415-435 . hal-00674821

\section{HAL Id: hal-00674821 \\ https://hal.science/hal-00674821}

Submitted on 24 Jun 2019

HAL is a multi-disciplinary open access archive for the deposit and dissemination of scientific research documents, whether they are published or not. The documents may come from teaching and research institutions in France or abroad, or from public or private research centers.
L'archive ouverte pluridisciplinaire HAL, est destinée au dépôt et à la diffusion de documents scientifiques de niveau recherche, publiés ou non, émanant des établissements d'enseignement et de recherche français ou étrangers, des laboratoires publics ou privés. 


\title{
Le simulateur automatisé BirthSIM pour la formation des obstétriciens
}

\author{
Richard Moreau ${ }^{1}$, Minh Tu PHAM ${ }^{1}$, Xavier BRUN $^{1}$, \\ Tanneguy REDARCE ${ }^{1}$, and Olivier DuPUIS ${ }^{2}$ \\ ${ }^{1}$ Laboratoire Ampère, UMR CNRS 5005, Université de Lyon, \\ INSA-Lyon, F-69621 Villeurbanne Cedex, France \\ ${ }^{2}$ Hospices Civils de Lyon (HCL), Hôpital Lyon-Sud, \\ CHU Lyon Sud, 69495 Pierre-Bénite, France
}

\begin{abstract}
Résumé
Cet article présente une des fonctionnalités du simulateur d'accouchement BirthSIM : la simulation d'accouchement instrumental. Son objectif est de proposer une nouvelle méthode de formation aux obstétriciens afin de compléter la formation traditionnelle. En obstétrique il peut être nécessaire d'utiliser des forceps pour extraire le nouveau-né, or ces instruments nécessitent une certaine expérience pratique. Cet article présente l'approche retenue pour simuler la dynamique d'un accouchement sur le simulateur BirthSIM. Nous nous intéressons particulièrement à une procédure qui permet de simuler un accouchement instrumental difficile en reproduisant les efforts mis en jeu lors d'un accouchement. Pour cela nous avons retenu une loi de commande originale : un asservissement en position avec un gain variable afin de modifier la raideur du système.
\end{abstract}

This paper presents one of the functionnality of the BirthSIM simulator : simulation of an instrumental delivery. Its aim is to offer a new kind of training for obstetricians in order to complete the traditionnal training. In obstetrics, forceps can be used to extract a fetus, these instruments require however a certain experience to be handled correctly. This paper explains the approach which allows to simulate the dynamic process of a childbirth. We especially focus on one procedure which reproduces a difficult instrumental delivery. We therefore decide to implement an original control law : a position feedback with a variable gain to regulate the stiffness of the system. The novice results emphasize the need of a childbirth simulator in order to gain initial experience without any risks.

Mots clés : Simulateur médical, commande, système mécatronique, système Fluid Power 


\section{Introduction}

À l'heure actuelle les obstétriciens acquièrent leur première expérience en salle $\mathrm{d}$ 'accouchement où ils appliquent leur savoir purement théorique. Cependant lorsque des complications surviennent (fœtus en situation d'hypoxie - manque d'oxygène - ou rythme cardiaque faiblissant), il est difficile d'apprendre correctement les bons gestes. En effet dans ce cas l'équipe médicale doit intervenir en urgence et utiliser des instruments obstétriques (forceps ou ventouse) pour extraire le fœtus. Apprendre dans ces conditions n'est pas idéal pour permettre aux jeunes obstétriciens d'acquérir une première expérience à cause des contraintes liées à de telles situations (stress, risque, urgence).

Dans cet article nous nous intéressons seulement à l'extraction par forceps car il s'agit de l'instrument obstétrique le plus utilisé dans les hôpitaux de Lyon et en France. La problématique liée à l'utilisation des forceps est l'apprentissage sans risque du geste. Cette problématique explique en partie la diminution de l'utilisation des forceps au profit de la ventouse ou de la césarienne alors qu'il s'agit de l'instrument le plus adapté pour assurer une naissance la plus physiologique possible. Le geste obstétrique lié à l'utilisation des forceps peut se décomposer en deux étapes : la pose des forceps et l'extraction. La pose des forceps a déjà fait l'objet de plusieurs études [1] [2] [3], le geste d'extraction n'a quant à lui été que très peu étudié.

Une formation sur simulateur permet de compléter la formation traditionnelle et offre la possibilité aux obstétriciens novices d'acquérir une expérience initiale. Un simulateur a donc été développé au laboratoire Ampère sous le nom BirthSIM. Celui-ci permet non seulement de s'entraîner à la pose des forceps mais aussi à l'extraction par forceps.

La principale contribution réside ici dans la description de l'algorithme de commande implémenté sur le simulateur pour reproduire des accouchements instrumentaux. Cet article se divise en quatre parties. La première est consacrée à la description des efforts mis en jeu pendant un accouchement. La seconde partie est dédiée à la description du simulateur BirthSIM, la génération de trajectoire et le réglage du correcteur permettant de reproduire les forces. Ensuite dans la troisième partie, le protocole expérimental et les premiers résultats expérimentaux sont présentés et enfin dans la dernière partie ces résultats sont discutés et les perspectives de travail présentées.

\section{Synchronisation des forces expulsives}

\subsection{Les forces expulsives naturelles}

L'objectif des forces expulsives naturelles fournies par la parturiente (femme en cours de travail d'accouchement) est de faire progresser le fotus dans le bassin maternel. Elles doivent donc vaincre les efforts résistifs des muscles pelviens qui tendent à retenir le fotus à l'intérieur. Les forces expulsives naturelles sont de deux types :

- les contractions utérines (CU) qui sont produites périodiquement et involontairement par la parturiente. Ces CU peuvent facilement être identifiées en utilisant un tocographe qui mesure les variations de pression intra-abdominale en fonction du temps [4]. Ces CU sont la source des forces expulsives automatiques (FEA) ;

- la pression abdominale que la parturiente exerce sur son utérus produit la force expulsive volontaire (FEV). Cette force est produite volontairement par la parturiente dans le but de compléter la FEA afin de vaincre la force résistive des muscles pelviens qui tendent à empêcher l'expulsion du fœtus. 
Des valeurs numériques de ces forces sont fournies par la littérature [5] [4]. Pendant la phase d'expulsion d'un accouchement, l'intensité totale d'une CU peut atteindre $9,7 \mathrm{kPa}$, ce qui correspond à $82 \mathrm{~N}$ pour une surface utérine moyenne de $85 \mathrm{~cm}^{2}$. Concernant l'intensité de la FEV, elle dépend surtout de la santé de la parturiente et peut atteindre $200 \mathrm{~N}$.

Une fonction gaussienne est le modèle le plus simple pour reproduire la forme des forces expulsives naturelles. Pour la FEA, cette fonction est de période $P$ pour assurer la simulation de plusieurs $\mathrm{CU}$ régulières. La fonction gaussienne utilisée, notée $\operatorname{FEA}(\mathrm{t})$, est définie par :

$$
F E A(t)=F E A(t+n P)=I\left(\exp \left(-\frac{t^{2}}{d}\right)\right)+T_{b}
$$

avec

- $n \in \mathbb{N}$ pour la périodicité de la fonction FEA;

- $t \in\left[t_{1}, t_{2}\right], t_{1}$ et $t_{2} \in \mathbb{R}^{2}$ définissent la période $P$ telle que $P=\left(t_{2}-t_{1}\right)$;

$-d \in \mathbb{R}^{+}$correspond à la durée de la FEA. En effet $d$ agit sur la largeur de la fonction gaussienne;

- $I \in \mathbb{R}^{+}$définit l'intensité vraie de la FEA i.e. son amplitude. Comme la fonction $\exp \left(-\frac{t^{2}}{d}\right)$ varie ente 0 et $1, I$ correspond à l'amplitude de la fonction qui est compris entre 60 et $100 \mathrm{~N}$ [5];

- $T_{b} \in \mathbb{R}^{+}$est le tonus de base de la $\mathrm{CU}$ et correspond à la valeur minimale entre deux CU.

La figure 1 montre la FEA simulée avec comme paramètres :

- une période $P$ de 180 secondes ;

- une durée $d$ de 60 secondes;

— une intensité $I$ de $80 \mathrm{~N}$;

— un tonus de base $T_{b}$ de $10 \mathrm{~N}$.

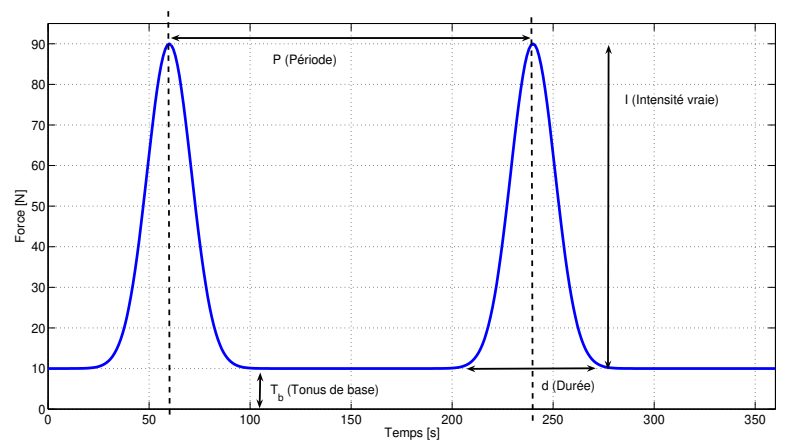

FIGURE 1 - Les différents paramètres de la FEA

La FEV est quant à elle définie par la fonction suivante :

$$
F E V(t)=I_{F E V}\left(\exp \left(-\frac{t^{2}}{d_{F E V}}\right)\right)
$$

Avec

$-t \in\left[t_{3}, t_{4}\right], t_{3}$ et $t_{4} \in \mathbb{R}^{2}$ sont prédéterminés mais peuvent être changés par l'instructeur, leurs valeurs correspondent à une durée de 50 secondes. Cette fonction n'est pas périodique et est déclenchée par l'instructeur sur la demande de l'opérateur;

$-d_{F E V} \in \mathbb{R}^{+}$est la largeur de la fonction gaussienne;

- $I_{F E V} \in \mathbb{R}^{+}$correspond à l'intensité de la FEV qui varie de 0 à $200 \mathrm{~N}$. 


\subsection{La force expulsive instrumentale (FEI)}

Pour certains accouchements la FEA et la FEV ne sont malheureusement pas suffisantes pour permettre l'extraction du fœetus, les obstétriciens doivent donc ajouter une force externe pour aider la progression du fotus. Cette force, appelée force expulsive instrumentale (FEI), est exercée par les obstétriciens à l'aide de leurs instruments (qui peuvent être des forceps ou une ventouse). L'objectif de cette FEI est de compléter la FEA et la FEV pour vaincre les efforts résistifs.

Il est à noter qu'il n'y a pas de valeurs précises dans la littérature pour cette force à cause de la difficulté de procéder à des mesures in vivo (d'un point de vue pratique et éthique). Cependant malgré ses difficultés, certains chercheurs ont essayé de quantifier cette force en instrumentant des forceps avec des capteurs de force. Les résultats obtenus sont dispersés et ne sont donc pas vraiment concluants. Selon l'instrumentation utilisée les résultats varient de 150 à $300 \mathrm{~N}$ que ce soit avec un dynanomètre [6], des jauges de contrainte [7] [8], des capteurs hydrogonflables [9] ou encore à partir de calculs théoriques reposant sur la pression maximale du liquide amniotique [10]. Plus de détails sont disponibles dans [11]. Une de nos contributions est de fournir une estimation réaliste de la FEI appliquée avec des forceps en utilisant un simulateur d'accouchement.

\subsection{Synchronisation des forces expulsives}

Afin de permettre l'extraction du fæetus, la somme des forces expulsives, notée force expulsive totale (FET), doit être supérieure à la force résistive qui tend à retenir le fœtus à l'intérieur du bassin. Ce principe conduit aux concepts de simple et double synchronisation. L'objectif est d'optimiser la FET afin d'assurer la progression du fœus tout en minimisant la FEI pour limiter les risques et ainsi obtenir un accouchement instrumental proche d'un accouchement eutocique (accouchement normal sans utilisation d'instruments obstétriques d'extraction). Quand deux forces expulsives sont concernées (FEA et FEV ou FEA et FEI), il s'agit d'une simple synchronisation. On parle de double synchronisation lorsque les trois forces sont concernées (cas le plus répandu lors d'accouchements instrumentaux). La figure 2 montre deux exemples de double synchronisation (une minimale et une parfaitement exécutée). Sur cette figure, la force résistive des muscles pelviens est fixée arbitrairement à $200 \mathrm{~N}$. Cette valeur peut être changée selon la difficulté de l'accouchement simulé.

\section{Simulation des forces mise en jeu pendant un accouchement}

\subsection{Description du simulateur BirthSIM}

Le simulateur BirthSIM est composé de trois parties (figure 3) :

- Une partie mécanique anthropomorphe (un bassin maternel et une tête fotale avec leurs principaux repères anatomiques) pour permettre à l'équipe médicale de retrouver des repères et des sensations haptiques proches de la réalité.

- Une partie électro-pneumatique pour reproduire la dynamique d'un accouchement. La partie électro-pneumatique est constituée d'un actionneur pneumatique et d'un servodistributeur pour simuler les différents efforts qui ont lieu pendant un accouchement.

- Une interface de visualisation qui fournit des informations complémentaires à l'équipe médicale comme la position des forceps à l'intérieur du bassin maternel et des informations sur les paramètres de l'accouchement simulé. 


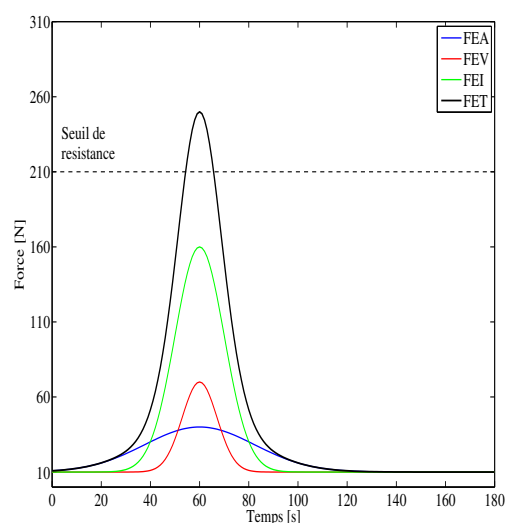

(a) très bonne synchronisation

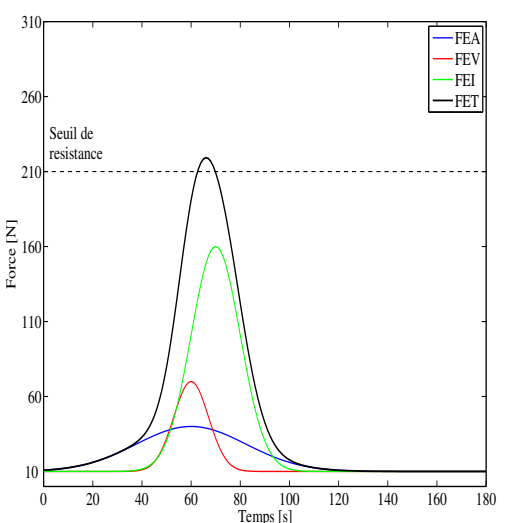

(b) synchronisation minimale

FIGURE 2 - Double synchronisation réussie : la FET est supérieure au seuil de résistance, le fœetus progresse dans le canal pelvien

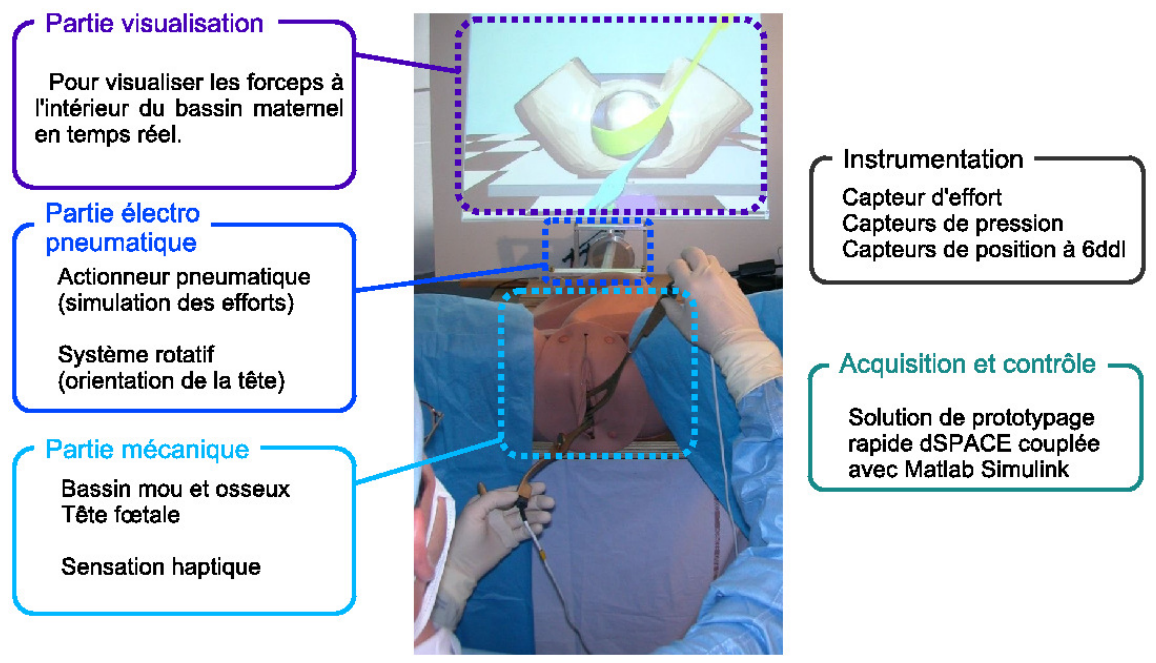

FIGURE 3 - Le simulateur BirthSIM

\subsection{La partie électro-pneumatique}

Dans cet article nous nous intéressons particulièrement à la partie électropneumatique qui permet de reproduire la dynamique d'un accouchement. Elle est composée (figure 4) :

- d'un vérin pneumatique;

- d'un servo-distributeur $5 / 3$ pour la régulation de débit;

- de deux capteurs de pressions montés près de chacune des chambres du vérin ;

- d'un capteur de position de type potentiomètre à câble ;

- d'un capteur d'effort en traction et compression monté entre la tête fœetale et l'extrémité du vérin.

La position de la tête fotale est repérée par rapport au plan défini par les épines sciatiques du bassin. Cette position est définie par l'ACOG (American College of Obstetrics and Gynecology) [12]. Dans cette classification, la position est appelée niveau et le niveau 0 correspond à la position pour laquelle le sommet de la tête est 


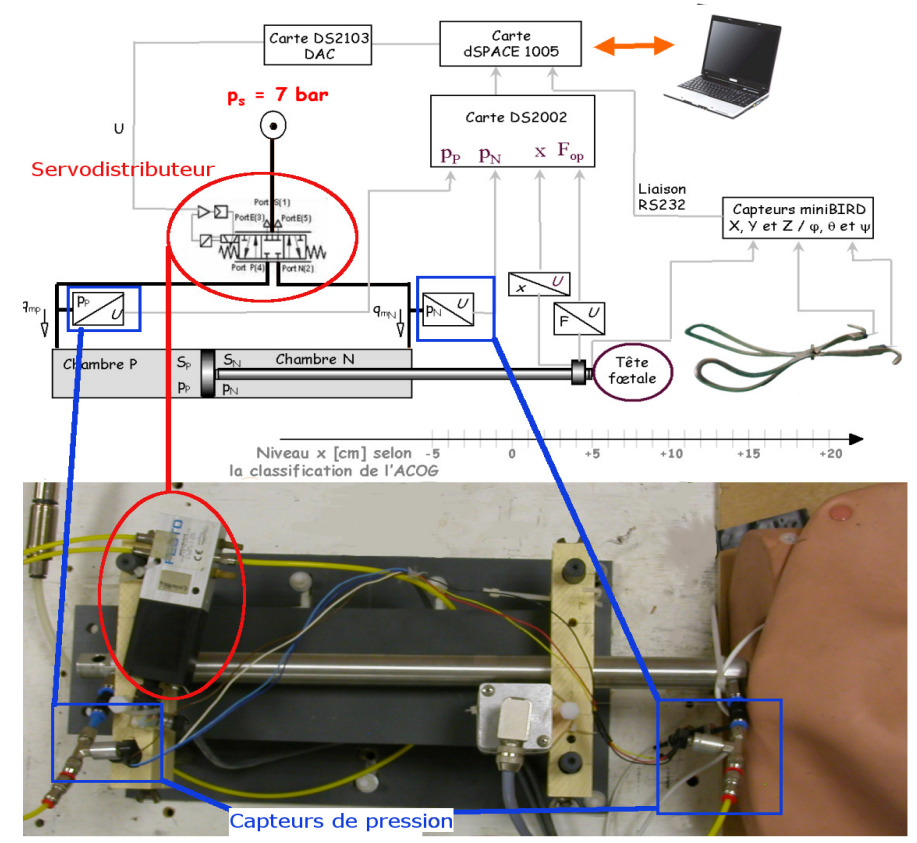

FIGURE 4 - Schéma de principe de la partie électro-pneumatique

tangent au plan transverse passant par les deux épines sciatiques.

La pression source $p_{s}$ est fixée à 7 bar absolus. Avec une telle source, le vérin pneumatique est capable de reproduire les différentes forces présentées dans la section précédente (section 2). Afin de simuler différents types d'accouchements, plusieurs procédures ont été mises en place sur le simulateur BirthSIM. Ces différentes procédures dédiées à la formation à l'extraction par forceps ont été présentées sans résultats expérimentaux dans [13] et la description des procédures lors d'un accouchement eutocique (accouchement sans complication) a été effectuée dans [14]. Il s'agissait d'un algorithme de commande qui commute entre un suivi de trajectoire en effort et une régulation de vitesse.

Le modèle de la partie électro-pneumatique est obtenu à partir des équations de trois phénomènes physiques : la loi de comportement du débit massique d'un gaz à travers une restriction, le comportement de la pression d'un gaz dans une chambre à volume variable et les équations mécaniques fondamentales. Le tableau 1 résume les principaux symboles et la nomenclature utilisés dans ce papier.

À partir des hypothèses classiques [15] ; [16] un modèle non linéaire du processus étudié peut être obtenu [17]. Ainsi en un point d'équilibre (dénoté avec l'exposant $e$ ), un modèle linéarisé tangent peut être établi à partir de ce modèle non linéaire [18] :

$$
d t\left[\begin{array}{l}
\delta p_{P} \\
\delta p_{N} \\
\delta v \\
\delta x
\end{array}\right]=\left[\begin{array}{cccc}
-\frac{1}{\tau_{P}^{e}} & 0 & -\frac{k p_{P}^{e} S_{P}}{V_{P}\left(x^{e}\right)} & 0 \\
0 & -\frac{1}{\tau_{N}^{e}} & \frac{k p_{N}^{e} S_{N}}{V_{N}\left(x^{e}\right)} & 0 \\
\frac{S_{P}}{M} & -\frac{S_{N}}{M} & -\frac{b_{v}}{M} & 0 \\
0 & 0 & 1 & 0
\end{array}\right]\left[\begin{array}{l}
\delta p_{P} \\
\delta p_{N} \\
\delta v \\
\delta x
\end{array}\right]+\left[\begin{array}{c}
\frac{k r T}{V_{P}\left(x^{e}\right)} G_{u_{P}}^{e} \\
-\frac{k r T}{V_{N}\left(x^{e}\right)} G_{u_{N}}^{e} \\
0 \\
0
\end{array}\right] \delta u
$$

Avec les constantes de temps $\tau_{P}^{e}$ et $\tau_{N}^{e}$ définies par :

$$
\tau_{P}^{e}=\frac{V_{P}\left(x^{e}\right)}{k r T C_{p_{P}}^{e}} \text { and } \tau_{N}^{e}=\frac{V_{N}\left(x^{e}\right)}{k r T C_{p_{N}}^{e}}
$$




\begin{tabular}{lll} 
Symbole & Description & Unité \\
\hline$b_{v}$ & Coefficient de frottement visqueux & {$[\mathrm{N} / \mathrm{m} / \mathrm{s}]$} \\
$C_{p}^{e}$ & $\begin{array}{l}\text { Dérivée partielle par rapport à } p \text { du } \\
\text { débit massique autour d'un point d'équilibre }\end{array}$ & {$[\mathrm{kg} / \mathrm{s} / \mathrm{Pa}]$} \\
$\delta X$ & Petite variation de X autour d'un point d'équilibre & \\
$F_{e x t}$ & Force externe & {$[\mathrm{N}]$} \\
$F_{f}$ & Force de frottement & {$[\mathrm{N}]$} \\
$F_{p r}$ & Force pneumatique & {$[\mathrm{N}]$} \\
$F_{S}$ & Force de stiction & {$[\mathrm{N}]$} \\
$G_{u}^{e}$ & Dérivée partielle par rapport à $u$ du & {$[\mathrm{kg} / \mathrm{s} / \mathrm{V}]$} \\
& débit massique autour d'un point d'équilibre & \\
$k$ & Constante polytropique & \\
$l$ & Longueur de la course du vérin & {$[\mathrm{m}]$} \\
$M$ & Masse totale en déplacement & {$[\mathrm{kg}]$} \\
$p_{X}$ & Pression dans la chambre X & {$[\mathrm{Pa}]$} \\
$q_{m}$ & Débit massique & {$[\mathrm{kg} / \mathrm{s}]$} \\
$r$ & Constante des gaz parfait & {$[\mathrm{J} / \mathrm{kg} / \mathrm{K}]$} \\
$S_{P}, S_{N}$ & Surfaces des pistons $P$ et $N$ & {$\left[\mathrm{~m}^{2}\right]$} \\
$T$ & Température ambiante & {$[\mathrm{K}]$} \\
$u_{P}, u_{N}$ & Tension des servo-distributeurs & {$[\mathrm{V}]$} \\
$V_{D X}$ & Volume mort de la chambre X & {$\left[\mathrm{m}^{3}\right]$} \\
$V_{X}$ & Volume de la chambre X & {$\left[\mathrm{m}^{3}\right]$} \\
$v$ & Vitesse & {$[\mathrm{m} / \mathrm{s}]$} \\
$x$ & Position & {$[\mathrm{m}]$}
\end{tabular}

TABLE 1 - Nomenclature

où

$$
\left\{\begin{array} { l } 
{ V _ { P } ( x ) = V _ { P } ( 0 ) + S _ { P } x } \\
{ V _ { N } ( x ) = V _ { N } ( 0 ) - S _ { N } x }
\end{array} \text { avec } \left\{\begin{array}{l}
V_{P}(0)=V_{D P}+S_{P} \frac{l}{2} \\
V_{N}(0)=V_{D N}-S_{N} \frac{l}{2}
\end{array}\right.\right.
$$

Et les coefficients de sensibilité du débit massique par rapport aux pressions $p$ et à la commande $u$ sont déduits de la caractérisation statique non linéaire globale du servo-distributeur FESTO [19] :

$$
\left\{\begin{array}{l}
C_{p_{P}}^{e}=-\left.\frac{\partial q_{m}\left(u^{e}, p_{P}\right)}{\partial p_{P}}\right|_{e_{1}}, \quad G_{u_{P}}^{e}=\left.\frac{\partial q_{m}\left(u, p_{P}^{e}\right)}{\partial u}\right|_{e_{1}} \\
C_{p_{N}}^{e}=-\left.\frac{\partial q_{m}\left(-u^{e}, p_{N}\right)}{\partial p_{N}}\right|_{e_{2}}, G_{u_{N}}^{e}=-\left.\frac{\partial q_{m}\left(-u, p_{N}^{e}\right)}{\partial u}\right|_{e_{2}}
\end{array}\right.
$$

\subsection{Algorithme de commande pour simuler un accouche- ment instrumental}

L'objectif de cet algorithme est de reproduire les différentes forces mises en jeu pendant un accouchement instrumental : les FEA et FEV mais aussi la force résistive des muscles pelviens. En plus de l'interface de visualisation où sont représentés en temps réel les positions des forceps, l'instructeur doit également avoir la possibilité de régler les différents paramètres de l'accouchement simulé : intensité et durée des FEA et des FEV, fréquence des FEA, etc.

Pendant la simulation d'un accouchement instrumental, les obstétriciens doivent exercer une force d'une intensité adaptée pour minimiser les risques. Dans cet article nous nous intéressons principalement à la procédure qui permet de reproduire une extraction par forceps difficile. Cette procédure simule le cas d'une parturiente 
fatiguée dont les forces naturelles (FEA et FEV) ne sont pas suffisantes pour expulser son fœtus. L'obstétricien doit alors utiliser des forceps pour extraire le fœtus.

Le réglage des paramètres de l'algorithme de commande a pour but de reproduire toutes les forces mises en jeu pendant un accouchement instrumental : FEA, FEV et aussi la force résistive qui empêchent la progression du fœetus. La première idée est d'utiliser un suivi de trajectoire en effort comme pour la simulation d'un accouchement eutocique. La force pneumatique de l'actionneur suit alors une consigne sur le modèle des contractions utérines présentées dans la soussection 2.1 et les forces résistives sont reproduites par les frottements mécaniques de l'actionneur. Malheureusement, ces forces de frottements ont été identifiées et se sont révélées trop faibles pour garantir un retour d'effort réaliste. L'idée retenue pour contourner ce problème est d'utiliser un autre algorithme simple et efficace : un asservissement en position avec un gain variable dans le temps pour contrôler la raideur du système. Ainsi l'actionneur pneumatique reproduit la somme des forces de la parturiente (FEA et FEV) et les forces résistives. La loi de commande associée est donc :

$$
U=K_{x}(t)\left(x_{d}-x\right)
$$

Le schéma correspondant est présenté sur la figure 5.

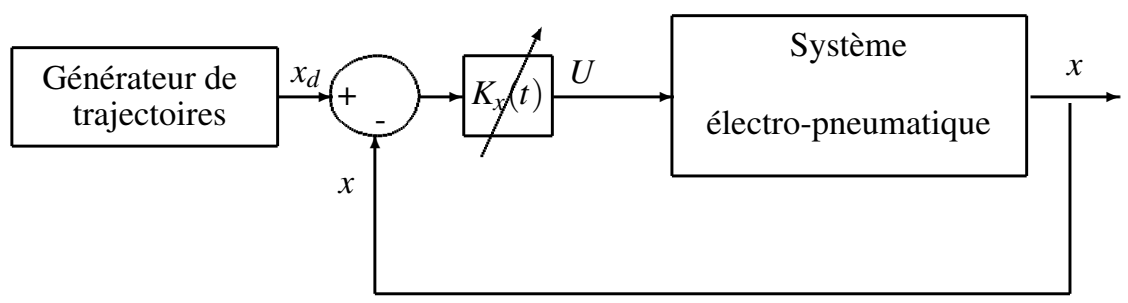

FIGURE 5 - Asservissement en position avec un gain variable

Les objectifs de cet algorithme est de reproduire des sensation haptiques réalistes et en particulier de reproduire le rôle des forces expulsives de la parturiente. L'originalité de cette loi de commande réside dans le fait que :

- $K_{x}$ n'est pas une constante mais est un paramètre variant avec le temps ;

- $x_{d}$ est la consigne en position dont le comportement est établi par un générateur de trajectoire prenant en compte la position mesurée de la tête fotale.

\subsubsection{Le générateur de trajectoire}

Pendant un accouchement, la tête fotale progresse par étape et peut être soumise à d'éventuels allers-retours si l'obstétricien n'applique pas une force suffisante pour la maintenir dans sa position. Pour simuler ce comportement, un générateur de trajectoire a été implémenté selon cet algorithme :

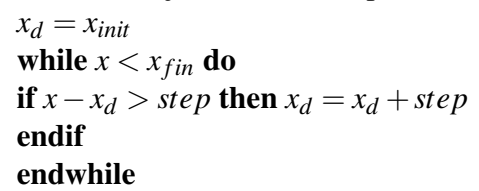

$x_{d}$ et $x_{\text {init }}$ sont respectivement la consigne de position et la position initiale de la tête fotale. L'extraction est considérée complète lorsque la position finale, $x_{\text {fin }}$, est atteinte. step est la valeur de l'incrément pour valider un déplacement à l'intérieur du bassin. Ces valeurs sont choisies par l'instructeur avant de réaliser les expériences. Par défaut, elles correspondent à $x_{\text {init }}=2 \mathrm{~cm}, x_{\text {fin }}=15 \mathrm{~cm}$ et $\mathrm{step}=$ $1 \mathrm{~cm}$. La figure 6 présente sous forme de schéma un exemple du comportement de 
$x_{d} \cdot x_{d}$ est incrémenté de la valeur de step si l'opérateur déplace la tête d'au moins cette valeur.

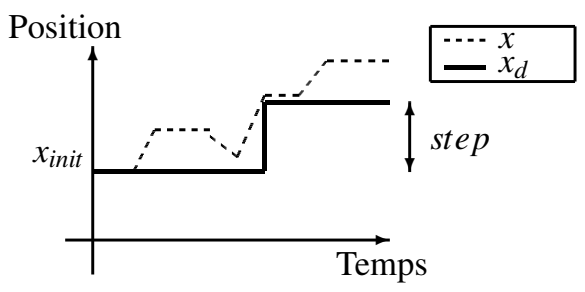

FIGURE 6 - Schéma d'un exemple du comportement de $x_{d}$ avec l'algorithme implémenté

\subsubsection{Réglage de la raideur de l'actionneur pneumatique}

La FEI nécessaire pour déplacer la tête fotale dépend du moment où l'obstétricien exerce son effort (concepts de synchronisation $c f$. sous-section 2.3). Pour reproduire ce phénomène, nous avons décidé de contrôler la raideur $K$ de l'actionneur pneumatique. Cette dernière est liée à la force de l'actionneur $F$ et à son déplacement $x$ par :

$$
K=\frac{\partial(\delta F)}{\partial(\delta x)}
$$

où la force de l'actionneur $F$ est :

$$
F=F_{p r}-F_{f}(v)-F_{\text {ext }}
$$

et :

$F_{p r}=S_{P} p_{P}-S_{N} p_{N}$ est la force pneumatique;

$F_{f}(v)=b_{v} v+F_{S} \operatorname{sgn}(v)$ est la force de frottement avec $\operatorname{sgn}(v)$ dépendant du signe de $v$ tel que : -1 si $v<0,1$ si $v>0$ et 0 si $v=0$;

- $F_{\text {ext }}=\left(S_{P}-S_{N}\right) p_{\text {atm }}$ est la force due à la pression atmosphérique $p_{a t m}$.

À partir du modèle linéarisé tangent [3] et en émettant l'hypothèse que la dynamique des pressions dans les chambres (de l'ordre de la seconde) peut être négligée par rapport à la dynamique d'une contraction utérine (de l'ordre de la minute) la relation entre la pression et la commande est donnée par :

$$
\left\{\begin{array}{l}
\delta p_{P}=\frac{G_{u_{P}}^{e}}{C_{P_{P}}^{e}} \delta u-\frac{p_{P}^{e} S_{P}}{r T C_{p_{P}}^{e}} \delta v \\
\delta p_{N}=-\frac{G_{u_{N}}^{e}}{C_{p_{N}}^{e}} \delta u+\frac{p_{N}^{e} S_{N}}{r T C_{P_{N}}^{e}} \delta v
\end{array}\right.
$$

Les gains en pression du servo-distributeur autour du point d'équilibre sont ainsi obtenus. Pour notre application les variations des pressions et de la commande sont très faibles, environ 0,35 bar et $50 \mathrm{mV}$ respectivement ( $c f$. résultats expérimentaux de la section 4.1 et la figure 4.1), les gains en pression peuvent ainsi être considérés comme constants. La variation de la force pneumatique peut donc s'écrire :

$$
\begin{aligned}
& \delta F_{p r}=S_{P} \delta p_{P}-S_{N} \delta p_{N} \\
& \delta F_{p r}=\left(S_{P} \frac{G_{u_{P}}^{e}}{C_{p_{P}}^{e}}+S_{N} \frac{G_{u_{N}}^{e}}{C_{p_{N}}^{e}}\right) \delta u-\left(\frac{p_{N}^{e} S_{N}^{2}}{r T C_{p_{N}}^{e}}+\frac{p_{P}^{e} S_{P}^{2}}{r T C_{p_{P}}^{e}}\right) \delta v
\end{aligned}
$$

La force externe, $F_{\text {ext }}$, est constante et la variation de la force de frottement est :

$$
\delta F_{f}(v)=b_{v} \delta v+\delta\left(F_{s} \operatorname{sgn}(v)\right)
$$


Lorsque la tête fœtale est extraite par l'obstétricien, elle se déplace dans le même sens (dirigé vers l'extérieur du bassin maternel) et donc $v$ garde toujours le même signe. Comme $F_{S}$ est constant, la variation $\delta\left(F_{S} \operatorname{sgn}(v)\right)$ est donc nulle. Lors du déplacement de la tête fortale, celle-ci peut être soumise à des déplacements irréguliers ce qui implique que $v$ peut changer de signe mais à ce moment là il n'y a plus de variation du frottement sec i.e. $F_{S}=0$ et donc $\delta\left(F_{S} \operatorname{sgn}(v)\right)$ est nul.

Ainsi à partir de [9], [11] et [12], nous pouvons écrire :

$$
\begin{aligned}
& \delta F=\delta F_{p r}-\delta F_{f}(v) \\
& \delta F=\left(S_{P} \frac{G_{u_{P}}^{e}}{C_{p_{P}}^{e}}+S_{N} \frac{G_{u_{N}}^{e}}{C_{p_{N}}^{e}}\right) \delta u-\left(\frac{p_{N}^{e} S_{N}^{2}}{r T C_{p_{N}}^{e}}+\frac{p_{P}^{e} S_{P}^{2}}{r T C_{p_{P}}^{e}}+b_{v}\right) \delta v
\end{aligned}
$$

La loi de commande est définie par l'équation [7] et pour une consigne constante, la relation entre la force et la position est :

$$
\delta F=-\left(S_{P} \frac{G_{u_{P}}^{e}}{C_{p_{P}}^{e}}+S_{N} \frac{G_{u_{N}}^{e}}{C_{p_{N}}^{e}}\right) K_{x} \delta x-\left(\frac{p_{N}^{e} S_{N}^{2}}{r T C_{p_{N}}^{e}}+\frac{p_{P}^{e} S_{P}^{2}}{r T C_{p_{P}}^{e}}+b_{v}\right) \delta v
$$

La raideur et les coefficients de frottements peuvent ainsi être identifiés, respectivement notés $K$ et $B$ :

$$
\begin{aligned}
& \delta F=-K \delta x-B \delta v \\
& K=\left(\begin{array}{c}
S_{P} G_{u_{P}}^{e} \\
\bar{C}_{p_{P}}^{e} \\
S_{N} G_{u_{N}}^{e} \\
C_{p_{N}}^{e}
\end{array}\right) K_{x} \\
& B=\left(\frac{p_{N}^{e} S_{N}^{2}}{r T C_{p_{N}}^{e}}+\frac{p_{P}^{e} S_{P}^{2}}{r T C_{p_{P}}^{e}}+b_{v}\right)
\end{aligned}
$$

La raideur du système en boucle fermée dépend donc de la variation du gain $K_{x}$. En effet la variation de la raideur peut simuler les variations des forces de la parturiente i.e. quand les forces expulsives maternelles augmentent, la raideur de notre système doit diminuer, et inversement quand les forces expulsives maternelles sont absentes, la raideur doit être élevée. Quand la tête fœtale est extraite $(\delta x>0)$ la variation de la force tend à maintenir la tête fotale à l'intérieur du bassin $(\delta F<0)$, la variation de la force est donc de signe opposé à la variation de la position.

Une des difficultés lors de la synthèse de la loi de commande est le réglage des paramètres car seule l'expérience des obstétriciens permet d'obtenir un retour d'effort réaliste lors de la simulation d'un accouchement instrumental.

Les paramètres de la fonction exponentielle de la fonction présentée en [1] et [2] sont donc définis empiriquement avec un expert obstétricien. Il doit exercer sa FEI comme il le ferait pendant un accouchement lorsque la FEV est absente puis lorsque celle-ci augmente. Ce réglage permet de déterminer l'amplitude de la fonction gaussienne selon son expérience clinique dans le but de lui permettre de retrouver des sensations haptiques proches de la réalité. Concernant le paramètre de durée, sa valeur est identique à la FEA et la FEV.

\section{Résultats expérimentaux}

\subsection{Validation du modèle}

Afin de valider les hypothèses retenus dans la section précédente (petites variations de la commande et des pressions), la loi de contrôle [7] a été implémentée sur le système. La figure 4.1 présente la commande $U$ appliquée au système et l'effort pneumatique $F_{p}$ mesurés pour un $K_{x}(t)$ donné. Cet effort correspond bien à la FEA mise en jeu lors d'un accouchement comme décrit en section 2.1.

En tenant compte du générateur de trajectoire implémenté (figure 6), $x$ est toujours supérieur ou égal à $x_{d}$. Ainsi, de [7] nous pouvons conclure que $\left(x_{d}-x\right)$ est toujours négatif et donc que la commande $U$ a un comportement opposé à $K_{x}$ comme le montre la figure 4.1. 

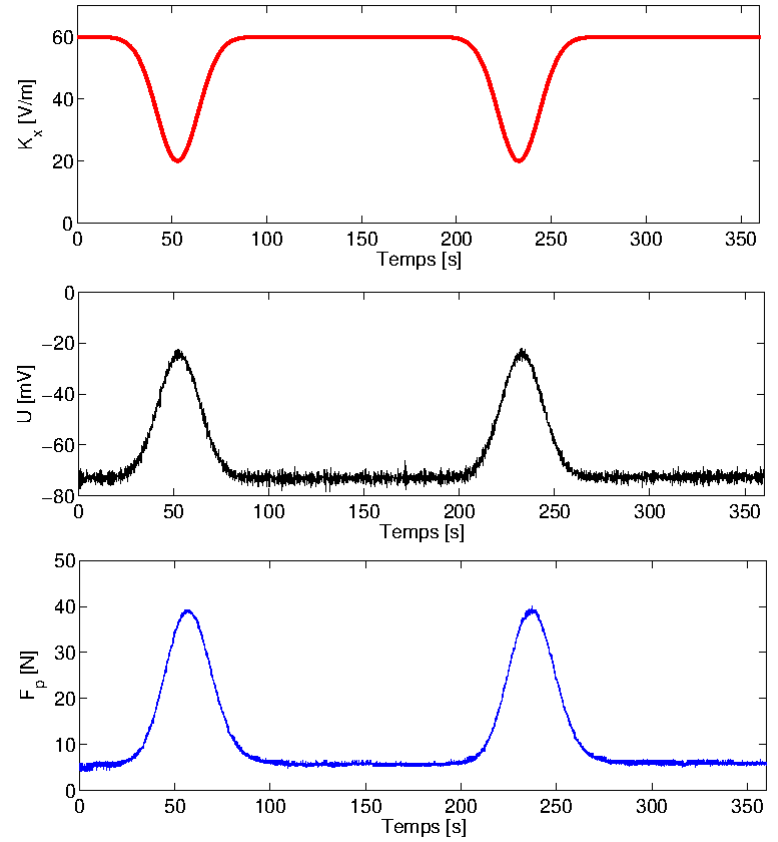

FiguRE 7 - Pour un $K_{x}(t)$ donné, $U$ et $F_{P}$ obtenus pour simuler une extraction par forceps difficile

\subsection{Protocole expérimental}

L'objectif du simulateur est d'offrir l'opportunité aux obstétriciens de se former sans risque à l'utilisation des forceps en leur permettant de se rendre compte des forces mises en jeu afin d'acquérir une première expérience en dehors de la salle d'accouchement. Un novice est un obstétricien avec moins de douze mois d'expérience obstétrique. Un expert est défini ici comme un obstétricien qui utilise les forceps comme instruments de prédilection lors de ses interventions instrumentées.

Rappelons que les forces mises en jeu sont en fait simulées par un gain $K_{x}$ variable représenté sur la figure 4.1. Les valeurs pour le générateur de trajectoires de la consigne en position sont : $x_{\text {init }}=2 \mathrm{~cm}, x_{\text {fin }}=15 \mathrm{~cm}$ et step $=1 \mathrm{~cm}$.

Les conditions initiales de tous les essais sont les suivantes :

- la présentation de la tête fœtale est $\mathrm{OP}+2$. OP signifie que la variété est occipitopubienne : les forceps doivent être placés symétriquement par rapport au bassin maternel. +2 signifie que le niveau correspond au niveau 2 i.e. que le sommet de la tête fotale est à $2 \mathrm{~cm}$ du plan des épines sciatiques : les forceps doivent donc être introduits relativement profondément dans le bassin maternel;

- la FEA est simulée par la variation de la raideur de l'actionneur pneumatique. La durée est de 60 secondes et la période est de 180 secondes;

- l'expérience est considérée terminée lorsque la tête foetale est complètement extraite de la vulve. Ceci correspond à une distance de $15 \mathrm{~cm}$ par rapport au plan des épines sciatiques. À partir de cette position l'obstétricien peut retirer ses forceps. Le choix de ces valeurs permet de définir les paramètres du générateur de trajectoire présentés dans la sous-section 3.3.1.

Ces conditions permettent de simuler le cas d'une parturiente considérée trop fatiguée pour extraire son fotus seule. Sa FEA n'est pas suffisante pour assurer la 
progression de son fotus et l'amplitude de la FEV qu'elle produit est faible. Les obstétriciens ont la possibilité de visualiser les forces simulées sur un écran afin de leur permettre de synchroniser leur FEI avec la FEA et la FEV simulée. Lors des premières expériences, deux novices ont participé à ces expériences et chacun a réalisé trois extractions par forceps. Nous nous intéressons ici seulement au geste d'extraction et nous assumons que la pose des forceps a été parfaitement réalisée. Leurs résultats sont comparés à ceux obtenus lors d'une extraction par forceps réalisée par un expert dans les mêmes conditions. Rappelons que dans cet article, seul le geste d'extraction est étudié, concernant l'analyse de la mise en place des forceps, celle-ci a été étudiée dans [11]; [20].

\subsection{Résultats et analyse}

La figure 8 présente la FEI appliquée par les novices lors de son extraction. Sur cette figure, la ligne pointillée représente la force expulsive maternelle (FEA + FEV) et les lignes pleines correspondent à la FEI appliquée par l'opérateur lors de ses différentes extractions. 


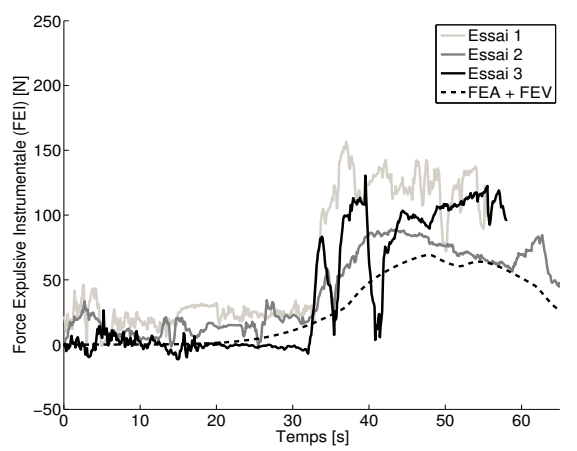

(a) novice 1

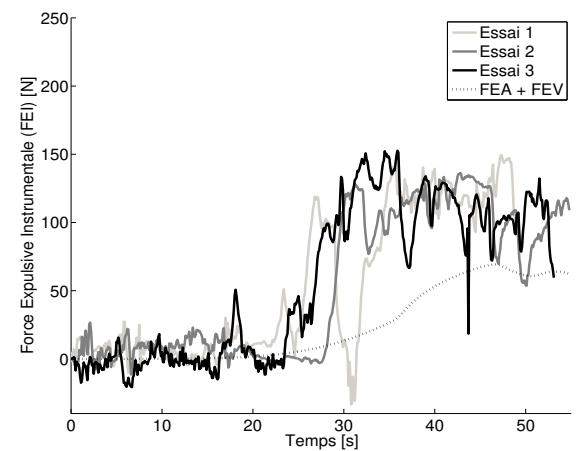

(b) novice 2

FIGURE 8 - FEI exercées par les novices lors d'une extraction simulée sur le simulateur BirthSIM

Outre le fait de proposer un entraînement sans risque, un simulateur offre également la possibilité de mesurer plusieurs paramètres afin d'évaluer un geste. Ainsi, en instrumentant la tête fotale avec un capteur de position il est possible de mesurer son déplacement lors de l'extraction. Les FEI représentées sur la figure 8 sont responsables des déplacements de la tête fœtale représentés sur la figure 9.

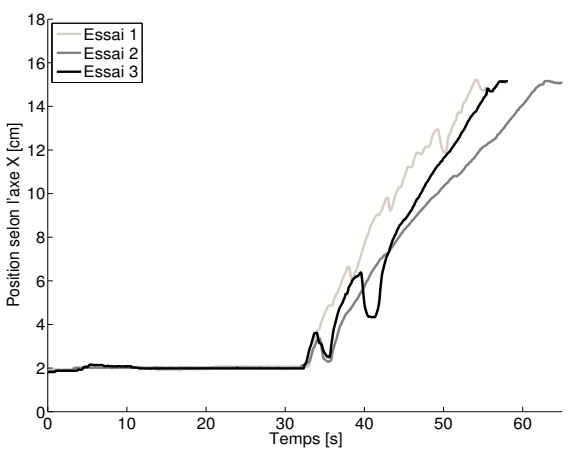

(a) novice 1

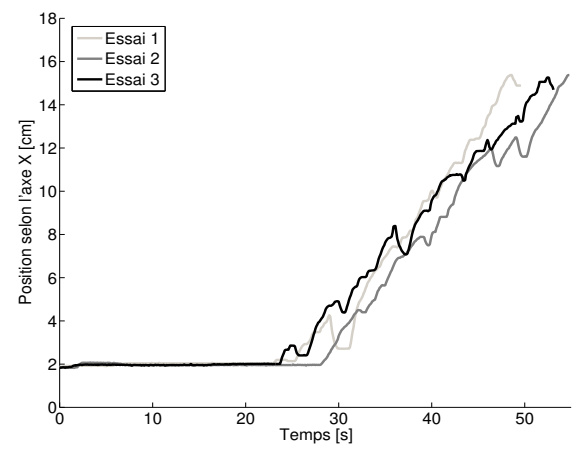

(b) novice 2

FIGURE 9 - Déplacements de la tête fotale dus aux FEI présentées sur la figure 8

Lors des extractions, les novices ont dû attendre que la FEA apparaisse pour commencer à synchroniser leur geste d'extraction. Les premières valeurs de la FEI sont dues à la mise en place des forceps qui peuvent provoquer des efforts sur la tête fœtale, mais ces efforts ne sont pas pris en compte dans l'analyse. Sur ces figures, la ligne pointillée représente les forces expulsives maternelles (FEA et FEV) et les lignes pleines correspondent à la FEA exercée par les novices lors de leurs différents essais.

Il apparaît sur ces figures que l'intensité maximale atteinte par la FEI est de $157 \mathrm{~N}$ pour le novice 1 et de $153 \mathrm{~N}$ pour le novice 2 . Concernant le déplacement de la tête fœtale (figure 9), elle a effectué un déplacement plus ou moins linéaire et a subit quelques allers-retours (surtout pour le novice 2) dans le bassin maternel qui peuvent avoir des conséquences dramatiques pour la parturiente et son enfant.

Concernant les résultats de l'expert, sa FEI et le déplacement de la tête fotale qui en découle sont représentés sur la figure 10.

Comme l'ont fait les novices, l'expert a attendu l'apparition de la première contraction utérine avant de procéder à l'extraction. Il est à noter que l'intensité de 


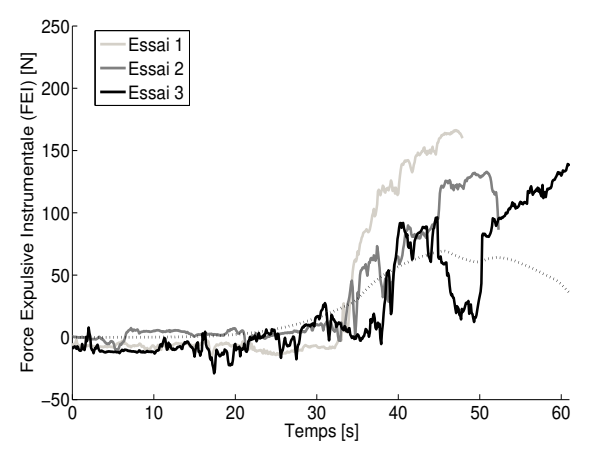

(a) FEI

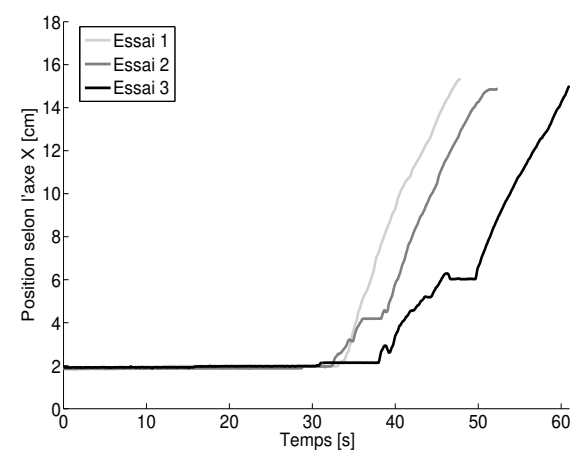

(b) Déplacement de la tête

FIGURE 10 - FEI exercées par un expert et déplacement de la tête correspondant

la FEI est moins élevée en moyenne que lors des essais des novices et que le déplacement de la tête est quasiment linéaire pendant toute l'extraction et n'effectue aucun aller-retour.

Afin de compléter cette analyse, les critères d'évaluation de la FEI reposent non seulement sur l'étude de son intensité maximale mais aussi sur son comportement dans le temps et l'espace, la moyenne et le travail de la FEI sont également pris en compte. Le tableau 2 rassemble tous les résultats. Ces résultats correspondent à la moyenne de chaque critère d'évaluation sur les trois essais. L'écart type est indiqué entre parenthèses.

\begin{tabular}{|c|c|c|c|}
\hline Critère & \multicolumn{3}{|c|}{ Essais d'extraction } \\
\cline { 2 - 4 } d'évaluation & Novice 1 & Novice 2 & Expert \\
\hline Travail de la FEI [J] & $16(5)$ & $19(2)$ & $13(2)$ \\
\hline FEI maximum [N] & $126(28)$ & $146(7)$ & $147(14)$ \\
\hline Moyenne de la FEI [N] & $47(10)$ & $54(2)$ & $34(2)$ \\
\hline Durée [s] & $61(4)$ & $54(2)$ & $55(6)$ \\
\hline
\end{tabular}

TABLE 2 - Résultats de la simulation d'extraction par forceps

La comparaison avec les résultats de l'expert montre que la FEI moyenne, le travail de la FEI et l'intensité maximale de la FEI ainsi que leur écart type respectif sont plus faibles pour l'expert. Le novice 1 a une FEI moyenne supérieure de $38 \%$ par rappport à l'expert (59\% pour le novice 2 ) et pour le travail de la FEI, le résultat du novice 1 est supérieur de $23 \%$ par rapport au résultat de l'expert (46\% pour le novice 2). Pour la durée, tous les opérateurs ont eu besoin approximativement du même temps pour extraire le fœtus. Cependant comme l'objectif de ces expériences est de permettre aux novices de se rendre compte des forces impliquées et non pas de simuler une procédure d'urgence, la durée n'est donc pas un paramètre déterminant. Concernant l'intensité maximale de la FEI, le résultat du novice 1 est plus faible que celui de l'expert mais cependant il est à noter que les novices exercent leur FEI avec une plus grande intensité plus longtemps que les experts (figures 8 et 10 ).

\section{Discussion}

En comparant ces résultats avec ceux de la littérature, l'ordre de grandeur de l'intensité de la FEI développée est le même. Les résultats présentés dans le ta- 
bleau 2 sont légèrement inférieurs à ceux de la littérature car la procédure utilisée simulait un accouchement par forceps « difficile »et non pas «très difficile ». Pour rappel, les résultats de la littérature proviennent de [6] et [21] qui montrent que les efforts maximum de traction se situent entre 150 et $300 \mathrm{~N}$. Ces mesures étaient réalisées in vivo en instrumentant des forceps avec un dynamomètre. Fleming et al. ont confirmé ces résultats en instrumentant des forceps avec des jauges de contraintes [7] puis Pearse les a utilisé pour démontrer que la force maximale moyenne se situe aux alentours de $190 \mathrm{~N}$ [21]. En 1966, Kelly a modifié légèrement la position des jauges de contraintes sur les forceps : ainsi à la place d'être situées sur la partie médiane des forceps, il les a placés sur l'extrémité des forceps qui sont en contact avec la tête fœtale. Dans [8] il a montré qu'au-delà de 230 N $50 \%$ des nouveau-nés sont victimes de lésions. De nos jours, la limite recommandée de la force d'extraction est fixée à $200 \mathrm{~N}$ [22].

La dispersion des résultats de la littérature confirme la difficulté de faire des mesures in vivo. En fait, il est difficile de mesurer l'effort réel de traction appliqué sur la tête fœala à cause de l'impossibilité d'instrumenter le fœtus avec des capteurs et de l'unicité de chaque accouchement. Les chercheurs peuvent donc aider les obstétriciens novices à appréhender la force à exercer en dehors de la salle d'accouchement. Leslie et al. ont ainsi proposé une formation à partir d'un bras mécanique sur lequel est attachée une paire de forceps instrumentée avec un capteur de force [23]. Dans notre cas, l'interface est anthropomorphe et les forceps ne sont pas attachés, ce qui permet d'assurer l'immersion des obstétriciens dans la simulation. Nos résultats aboutissent à la même conclusion : les obstétriciens novices ont besoin de s'entraîner pour prendre conscience des forces qu'ils exercent et cette formation n'est jamais évidente à réaliser pendant un véritable accouchement. De plus, les résultats présentés dans cet article montrent que les résultats des novices sont très dispersés ce qui signifie que la formation doit être personnalisée. Or ceci n'est pas possible pendant la formation traditionnelle alors qu'une formation sur simulateur le permet. Ces premiers résultats sont plutôt encourageants mais nécessitent d'être confirmés par une plus grande campagne de mesure.

Le capteur de force utilisé sur le simulateur BirthSIM est seulement utilisé en tant qu'instrument de mesure. Nous ne l'avons pas utilisé dans l'optique d'une commande en effort car nous voulions d'abord vérifier que nos résultats étaient proches de ceux de la littérature. De plus, un asservissement en position permet de reproduire un déplacement réaliste de la tête fotale qui tend à être retenue à l'intérieur du bassin par les muscles pelviens, ce qui se traduit par des déplacements saccadés si l'obstétricien n'exerce pas une force pour maintenir la tête.

Un des avantages majeur d'un simulateur est de proposer non seulement un entraînement sans risque, mais aussi de permettre l'étude de différents paramètres qui sont difficiles voire impossibles à mesurer lors d'accouchement comme par exemple le déplacement de la tête fotale et la force réelle appliquée sur la tête. Un simulateur peut ainsi aussi être utilisé comme un outil de recherche pour les obstétriciens pour comparer et valider de nouvelles techniques et de nouveaux instruments.

\section{Conclusion}

La stratégie de commande présentée et implémentée dans la partie électropneumatique du simulateur BirthSIM répond aux besoins des obstétriciens. Différents scénarios sont disponibles sur le simulateur BirthSIM. Dans cet article, nous nous sommes intéressés à la simulation d'accouchements difficiles par forceps. La loi de commande est un asservissement en position avec un gain $K_{x}$ variable qui permet de modifier la raideur du système et de reproduire les différentes forces qui 
ont lieu pendant un accouchement. Nous profitons ainsi d'une des caractéristiques des systèmes pneumatiques que n'ont pas les systèmes électriques : le contrôle de la compliance. L'objectif de ces expériences était de permettre aux novices de prendre conscience des forces mises en jeu.

Leurs gestes d'extraction ont ensuite été comparés avec ceux d'un expert. Cette comparaison a permis de conclure que les novices ont besoin d'acquérir une expérience permettant d'optimiser la FEI qu'ils appliquent sur la tête fotale. Ces premiers résultats permettent de montrer que les novices ont besoin d'un entraînement afin de maîtriser leur force. De plus chaque novice obtient des résultats différents, les étudiants ont donc besoin d'un entraînement personnalisé pour leur permettre d'acquérir de l'expérience. Cette personnalisation de l'entraînement n'est pas possible en salle d'accouchement lors de la formation traditionnelle mais elle peut être effectuée en complément de cette formation sur un simulateur d'accouchement. Cette première expérience permet aux novices d'avoir une plus grande confiance en soi et d'être techniquement prêt le jour où un accouchement instrumental a lieu. Les valeurs obtenues pendant les expériences sur simulateur sont du même ordre de grandeur que ceux de la littérature, ce qui garantit une formation réaliste sur simulateur.

Le déplacement de la tête fotale lors de l'extraction a également été étudié. Nous avons pu ainsi montrer que, selon la force exercée, la tête fotale peut être soumise à des allers-retours très préjudiciables pour la santé de la mère et de son fœtus. En maîtrisant mieux la FEI qu'ils exercent, les obstétriciens peuvent limiter ces allers-retours et assurer une extraction plus linéaire. Un des objectifs était aussi de leur montrer que certains d'entre eux peuvent aisément dépasser la limite recommandée de $200 \mathrm{~N}$ et de leur permettre de se rendre compte de la force qu'ils peuvent exercer et des conséquences de cette force sur l'extraction du fœtus.

Un programme de formation est actuellement en cours à l'hôpital Lyon-Sud des Hospices civils de Lyon pour permettre aux novices d'améliorer leurs gestes et de procéder à des accouchements par forceps sans risque sous la supervision d'un expert.

\section{Références}

[1] R. Lapeer, M. S. Chen, and J. Villagrana. An augmented reality based simulation of obstetric forceps delivery. In $3^{\text {rd }}$ IEEE and ACM International Symposium on Mixed and Augmented Reality, (ISMAR'04), pages 274-275, 2-5 Nov. 2004.

[2] T. Sielhorst, T. Obst, R. Burgkart, R. Riener, and N. Navab. An augmented reality delivery simulator for medical training. In Augmented environments for Medical Imaging including Augmented Reality in Computer-aided Surgery (AMI ARCS'04), pages 11-20, Rennes, France, 2004.

[3] R. Moreau, M.T. Pham, T. Redarce, and O. Dupuis. A new learning method for obstetric gestures using the birthsim simulator. In IEEE International Conference on Robotics and Automation (ICRA'07), pages 2279-2284, Roma, Italy, 10-14 April 2007.

[4] E. Papiernik, D. Cabrol, and J.-C. Pons. Obstétrique. Flammarion MédecineSciences, 1997. ISBN 2257150457.

[5] J.-M. Thoulon. Le monitorage électronique foctal : la cardiotocographie. 2e ed., Masson, 1991.

[6] B. Wylie. Traction in forceps deliveries. American Journal of Obstetrics and Gynecology (AJOG), 20 :425-433, 1933. 
[7] A. R. Fleming, K. R. Brandeberry, and W. H. Pearse. Introduction of a metric forceps. American Journal of Obstetrics and Gynecology (AJOG), 78 :125133, 1959.

[8] J. V. Kelly and G. Sines. An assessment of the compression and traction forces of obstetrical forceps. American Journal of Obstetrics and Gynecology (AJOG), 96 :521-537, 1966.

[9] A. Moolgoaker. A new design of obstetric forceps. Obstetrics and Gynecology, 69 :450-457, 1962.

[10] P. Rosa. Defense of suction grip extraction. Brux Med, 35 :1590-1597, 1955.

[11] R. Moreau, M.T. Pham, R. Silveira, T. Redarce, X. Brun, and O. Dupuis. Design of a new instrumented forceps : Application to safe obstetrical forceps blade placement. IEEE Transactions on Biomedical Engineering, 54(7) :1280-1290, July 2007.

[12] G. Cunningham, L. Gilstrap, K. Leveno, S. Bloom, J. Hauth, and K. Wenstrom. Williams Obstetrics. the McGraw-Hill Companies, 22 ${ }^{\text {nd }}$ edition, 2005.

[13] O. Olaby, R. Moreau, X. Brun, O. Dupuis, and T. Redarce. Automatic childbirth procedures implanted on the BirthSIM simulator. In IEEE/RSJ International Conference on Intelligent Robots and Systems (IROS'06), pages 23702375, Beijing, China, 2006.

[14] R. Moreau, O. Olaby, M.T. Pham, X. Brun, and O. Dupuis. Simulateur d'accouchement automatisé BirthSIM pour l'apprentissage sans risque des gestes obstétricaux. In Journées Doctorales MACS - JDMACS'07, Reims, France, 2007.

[15] B. L. Andersen. The Analysis and Design of Pneumatic Systems. Wiley, New-York, 1967.

[16] J. L. Shearer. Study of pneumatic processes in the continuous control of motion with compressed air. parts I and II. Trans. Am. Soc. Mech. Eng., $78: 233-249,1956$.

[17] X. Brun, D. Thomasset, and E. Bideaux. Influence of the process design on the control strategy : application in electropneumatic field. Control Engineering Practice, 10(7) :727-735, July 2002.

[18] X. Brun, S. Sesmat, D. Thomasset, and Scavarda S. A comparative study between two control laws of an electropneumatic actuator. In European Control Conference (ECC'99), pages F1000-5, Karlsruhe, Germany, Sept. 1999.

[19] O. Olaby, S. Sesmat, X. Brun, T. Redarce, and E. Bideaux. Characterization and modelling of a FESTO proportionnal valve in aim of control synthesis. In The $6^{\text {th }}$ JFPS International Symposium on Fluid Power, Tsubaka, Japan, November 7-10 2005.

[20] R. Moreau, V. Ochoa, M.T. Pham, P. Boulanger, T. Redarce, and O. Dupuis. A method to evaluate skill transfer and acquisition of obstetric gestures based on the curvatures analysis of the position and the orientation. Elsevier, Journal of Biomedical Informatics, 41 :991-1000, 2008. doi :10.1016/j.jbi.2008.03.012.

[21] W. H. Pearse. Electronic recording of forceps delivery. American Journal of Obstetrics and Gynecology (AJOG), $86: 43-51,1963$.

[22] P.C. Dennen. Dennen's Forceps Deliveries. F.A. Davis, $3^{\text {rd }}$ ed. edition, 1989. ISBN : 0803625111.

[23] K. Leslie, P. Dipasquale-Lehnerz, and M. Smith. Obstetrics forceps training using visual feedback and the isometric strength testing unit. Obstetrics \& Gynecology, $105: 377: 382,2005$. 